\title{
RELATIONS AMONG CHARACTERISTIC CLASSES
}

\author{
BY \\ STAVROS PAPASTAVRIDIS( ${ }^{(1)}$
}

\begin{abstract}
Let $M$ be an $n$-dimensional, compact, closed, $C^{\infty}$ manifold, and $\nu: M \rightarrow B O$ be the map classifying its stable normal bundle. Let $S \subseteq$ $H^{*}\left(B O ; Z_{2}\right)$ be a set of characteristic classes and let $q, k$, be fixed nonnegative integers. We define $I_{n}^{q}(S, k)=\left\{x \in H^{q}(B): \nu^{*}(x) \cdot y=0\right.$ for all $y \in H^{k}\left(M ; Z_{2}\right)$ and for all $n$-dimensional, $C^{\infty}$ closed compact manifolds $M$, which have the propery that $\left.\nu^{*}(S)=\{0\}\right\}$.

In this paper we compute $I_{n}^{q}(S, k)$, where all classes of $S$ have dimension greater than $n / 2$. We examine also the case of $B S O$ and $B U$ manifolds.
\end{abstract}

0. Introduction. It is well known that certain characteristic classes are zero on the normal bundles of all $C^{\infty}$ manifolds of a given dimension, although they are not zero for all bundles. The basic reference is the work of $\mathrm{E}$. $\mathrm{H}$. Brown and F. P. Peterson (see [2], [3], [4]). In this paper we are going to study some aspects of this phenomenon.

First of all let us settle the terminology. Throughout this paper, unless otherwise stated, $B$ will be the classifying space for some bundle theory, i.e. $B O, B S O, B$ Spin, etc., $\gamma$ will be its universal bundle and $p$ is a prime number such that $\gamma$ is orientable and oriented in $Z_{p}$ coefficients, namely a Thom class $U \in H^{m}\left(T(\gamma) ; Z_{p}\right)$ is given. Let $S \subseteq H^{*}\left(B ; Z_{p}\right)$ be a set of characteristic classes in our theory. From now on unless otherwise stated all cohomology groups will have coefficient group $Z_{p}$.

Definition 0.1. A $B-S$ manifold of dimension $n$ is a couple $(M, v)$ where $M$ is an $n$-dimensional, compact, closed, connected, $C^{\infty}$ manifold and $\nu$ : $M \rightarrow B$ is a map such that the pull-back of $\gamma$ under $\nu, \nu^{*}(\gamma)$, is isomorphic with the stable normal bundle of $M$, and furthermore $\nu^{*}(S)=\{0\}$. A $B-\varnothing$ manifold will be called simply a $B$ manifold.

Definition 0.2. We define $I_{n}^{q}(B, S, p, k)=\left\{x \in H^{q}(B): \nu^{*}(x) \cdot y=0\right.$ for all $y \in H^{k}(M)$ and for all $n$-dimensional $B$-S manifolds $\left.(M, \nu)\right\}$, where $q, k$ are nonnegative integers such that $q+k \leqslant n$. We call $I_{n}^{q}(B, S, p, k)$ the set

Received by the editors July 22, 1976.

AMS (MOS) subject classifications (1970). Primary 57D20, 57D75, 57D90; Secondary 55H10, 57D65.

Key words and phrases. Characteristic classes, characteristic numbers, corbodism, surgery, Steenrod algebra, Serre spectral sequence.

(')Research partially supported by NSF Grant GP-38920X.

O American Mathematical Society 1978 
of relations among $q$ dimensional mod- $p$ characteristic classes, in $n$-dimensional $B-S$ manifolds.

From now on throughout this paper, $q, n, k$ will be fixed nonnegative integers such that $q+k \leqslant n$. The set $I_{n}^{q}(B, \varnothing, p, 0)$ is Brown-Peterson's $I_{n}^{q}(B, p)$ (see [2, p. 39]). In this paper we are going to study the set $I_{n}^{q}(B, S, p, k)$ (actually it is a $Z_{p}$ space). Specifically, we are interested in studying the following two questions:

(a) compare $I_{n}^{q}(B, S, p, k)$ and $I_{n}^{q}(B, \varnothing, p, k)$,

(b) compare $I_{n}^{q}(B, \varnothing, p, k)$ and $I_{n}^{q}(B, \varnothing, p, 0)$.

In order to be able to express our result in a more conceptual way, we find it convenient to introduce an algebraic analogue of $I_{n}^{q}(B, S, p, k)$. In doing so we follow some ideas of J. F. Adams (see [1]) and Brown-Peterson (see [4]). But in order to do that we need some preparation.

Definition 0.3. We call $H$ an $n$-dimensional Poincare algebra if $H$ is a graded algebra with unit which is associative and commutative (in the graded sense) and furthermore $H$ is a left module over $A$ (the mod- $p$ Steenrod algebra) in the graded sense, which satisfies the following conditions:

(a) The action of $A$ satisfies the Cartan formula, namely if $\psi: A \rightarrow A \otimes A$ is the well-known diagonal in the Steenrod algebra (see [8, p. 150]) and $\psi(a)=\sum a^{\prime} \otimes a^{\prime \prime}$ and $x, y \in H$ then

$$
a(x y)=\sum:(-1)^{\operatorname{deg} x \cdot \operatorname{deg} a^{\prime \prime}} a^{\prime} x \cdot a^{\prime \prime} y .
$$

(b) The action of $A$ is unstable, namely if $p=2$ then $\mathrm{Sq}^{i} x=0$ if $i>\operatorname{dim} x$, and $\mathrm{Sq}^{i}\langle x\rangle=x^{2}$ if $i=\operatorname{dim} x$, and if $p \neq 2$ then $P^{i} x=0$ if $2 i>\operatorname{dim} x$ and $P^{i} x=x^{p}$ if $2 i=\operatorname{dim} x$.

(c) $H^{i}=0$ for $i<0$ or $i>n$.

(d) An element $m_{H} \in\left(H^{n}\right)^{*}=\operatorname{Hom}\left(H^{n}, Z_{p}\right)$ is given (it is part of the structure of a Poincaré algebra) so that the bilinear map $H^{i} \times H^{n-i} \rightarrow Z_{p}$ defined by $(x, y) \rightarrow m_{H}(x \cdot y)$ for $x \in H^{i}, y \in H^{n-i}$, for all $0 \leqslant i \leqslant n$, is nonsingular.

It is clear that the concept of Poincare algebra is the abstraction of the algebraic properties of the $Z_{p}$ cohomology of an orientable manifold.

Following Brown-Peterson we introduce a right $A$ action on $H^{*}(B)$, using the bundle $\gamma$ as follows: let $U \in H^{*}(T(\gamma))$ be the Thom class of $\gamma(\gamma$ was assumed $Z_{p}$-oriented), then if $a \in A, x \in H^{*}(B) x \cdot a$ if defined by the equality

$$
x a \cdot U=\chi(a)(x U)
$$

where $\chi$ is the well-known canonical antihomomorphism of $A$ (see [8, p. 166]). This right action of $A$ on $H^{*}(B)$ just makes $H^{*}(B)$ a right module over $A$, it does not have any particular properties like the Cartan formula or something like that. In exactly the same way we can define a right action on the $Z_{p}$ 
cohomology of any $Z_{p}$-oriented $C^{\infty}$ manifold by using its stable normal bundle. In connection with that, J. F. Adams observed (see [1, p. 742]) that a right $A$ action on the $Z_{p}$ cohomology of a differentiable $Z_{p}$-oriented manifold can be defined from the algebra structure without any reference to the normal bundle.

Specifically if $H$ is an $n$-dimensional Poincaré algebra and $x \in H^{i}, a \in A_{j}$ then $x \cdot a \in H^{i+j}$ is defined by the property that

$$
m_{H}(x a \cdot y)=m_{H}(x \cdot a y) \text { for all } y \in H^{n-i-j} .
$$

In the case that $H$ is the $Z_{p}$ cohomology of a $Z_{p}$-oriented manifold, it can be proved that the right $A$ action introduced by $(0.5)$ is the same with the action defined by (0.4) via the stable normal bundle of the manifold (for a proof of that see [2, p. 46, Corollary 6.3]), so if $(M, \nu)$ is a $B$ manifold, then $\nu^{*}: H^{*}(B) \rightarrow H^{*}(M)$ is a map of algebras and $A$ left-right modules.

From now on throughout this paper all Poincaré algebras will be considered as right $A$ modules, with the action defined via (0.5), and the $Z_{p}$ cohomology of all spaces with a distinguished bundle $Z_{p}$-oriented, will be considered a right $A$ module with the action defined via (0.4).

Definition 0.6. An $n$-dimensional $B-S$ Poincaré algebra, is a couple $(H, v)$, where $H$ is an $n$-dimensional Poincare algebra and $\nu: H^{*}(B) \rightarrow H$ is a map of a graded algebras and right-left modules over $A$, such that $\nu(S)=$ $\{0\}$. A $B-\varnothing$ Poincaré algebra will be called simply a $B$ Poincaré algebra.

We define alg $I_{n}^{q}(B, S, p, k)=\left\{x \in H^{q}(B): \nu(x) \cdot y=0\right.$ for all $y \in H^{k}$ and for all $n$-dimensional $B-S$ Poincaré algebras $(H, v)\}$.

Obviously if $(M, \nu)$ is a $B-S$ manifold then $\left(H^{*}(M), \nu^{*}\right)$ is a $B$-S Poincare algebra.

If $Y \subseteq H^{*}(B)$ then [Y] will be the smallest subset of $H^{*}(B)$ which contains $Y$, it is an ideal of $H^{*}(B)$ and it is closed under the left action of $A$. It can be proved easily from $(0.4)$ that $[Y]$ is closed under the right action of $A$. Let us call $i_{Y}$ the inclusion $Y \rightarrow H^{*}(B)$.

From the Eilenberg-Mac Lane space $K\left(Z_{p}, j\right)$ let $c_{j}$ be its fundamental class, for $j>0$. We agree that $K\left(Z_{p}, 0\right)=*$ and $c_{0}=1 \in H^{0}(*)$. Let $X=$ $K\left(Z_{p}, k\right) \times K\left(Z_{p}, n-k-q\right)$ and let $l: H^{*}(B) \rightarrow H^{*}(B) \otimes_{A} H^{*}(X)$ be the map defined by the formula $l(x)=x \otimes_{A} c_{k} \otimes c_{n-q-k}$. Observe that $l$ increases degree by $(n-q)$.

Now we are ready to state our main results.

THEOREM 0.7. (a) alg $I_{n}^{q}(B, S, p, k)$ consists of those elements $x \in H^{q}(B)$ such that $l(x) \in \operatorname{image}\left(i_{[S]} \otimes_{A} I\right)\left(I\right.$ is the identity on $\left.H^{*}(X)\right)$.

(b) Let $d$ be the minimum dimension of elements of $S$, and suppose that $d>n / 2$. If $p=2$ and $d+n+1>2 q$ or if $p \neq 2$ and $d+n>2 q$, then:

$$
I_{n}^{q}(B, S, p, 0)=I_{n}^{q}(B, \varnothing, p, 0)+[S] \text {. }
$$


THEOREM 0.8. We assume that $d>n / 2$. Then if $p=2$ and $B=B O$ or $B U$, or if $p \neq 2$ and $B=B S O$ or $B U$ we have:

(a) $I_{n}^{q}(B, S, p, k)=\operatorname{alg} I_{n}^{q}(B, S, p, k)$,

(b) $I_{n}^{q}(B, \varnothing, p, k)=I_{n}^{q}(B, \varnothing, p, 0)$.

The organization of the paper is as follows. In $\$ 1$ we outline a general technique for studying these problems and we prove Theorem 0.7. In \$2 we prove Theorem 0.8 for $B=B O$ and in $\$ 3$ we prove Theorem 0.8 for the case $B=B S O$ or $B U$. In a future paper we plan to examine the case $p=2$, $B=B S O$.

The basic ideas of this paper are due to E. Brown and F. Peterson. I am indebted to both of them for many illuminating discussions.

1. General considerations and outlines of the general technique. Following Brown-Peterson, let $\Omega_{n}(Y, B)=\pi_{n+m}\left(T(\gamma) \wedge Y^{+}\right)$be the $B$-bordism group of a space $Y$ and let $\Omega_{n}^{*}(Y, B)=\operatorname{Hom}\left(\Omega_{n}(Y, B) ; Z_{p}\right)$ be its dual. $Y$ is a $C W$ complex with a finite number of cells in it its dimension, $Y^{+}=Y U\{*\}$, and $m$ is the dimension of the bundle $\gamma$, we can assume that $m>4 n$. As in [2, pp. 41-42] there is a natural map $\theta:\left[H^{*}(B) \otimes_{A} H^{*}(X)\right]^{n} \rightarrow \Omega^{*}(X ; B)$. From now on, we assume that the elements of $S$ are linearly independent over $Z_{p}$, clearly Theorems $0.7,0.8$ will not be affected. Next we define a map

$$
B \rightarrow \prod_{s \in S} K\left(Z_{p}, \operatorname{dim} s\right)
$$

so that the pull-back of the fundamental class of $K\left(Z_{p}, \operatorname{dim} s\right)$ is $s \in H^{*}(B)$. Then the standard way we consider the fibration of paths over $\Pi_{s \in S} K\left(Z_{p}, \operatorname{dim} s\right)$ and we pull it back over $B$, so we get a fibration $\pi$ : $B^{\prime} \rightarrow B$ with fibre $\Pi_{s \in S} K\left(Z_{p}\right.$, dim $\left.s-1\right)$. Let $\gamma^{\prime}=\pi^{*}(\gamma)$ be the pull-back of $\gamma$ over $B^{\prime}$. Then we can define the $B^{\prime}$-bordism groups as $\Omega_{n}\left(Y, B^{\prime}\right)=$ $\pi_{n+m}\left(T\left(\gamma^{\prime}\right) \wedge Y^{+}\right)$, and the relative $\left(B, B^{\prime}\right)$-bordism groups as $\Omega_{n}\left(Y, B, B^{\prime}\right)$ $=\pi_{n+m}\left(T(\gamma) / T\left(\gamma^{\prime}\right) \wedge Y^{+}\right)$(for details see [12, p. 25]). We can make $H^{*}\left(B^{\prime}\right), H^{*}\left(B, B^{\prime}\right)$ right $A$ modules in exactly the same way as in (0.4), and we can define maps

$$
\begin{gathered}
\theta^{\prime}:\left[H^{*}\left(B^{\prime}\right) \otimes_{A} H^{*}(X)\right]^{n} \rightarrow \Omega_{n}^{*}\left(X ; B^{\prime}\right), \\
\theta^{\prime \prime}:\left[H^{*}\left(B, B^{\prime}\right) \otimes_{A} H^{*}(X)\right]^{n} \rightarrow \Omega_{n}^{*}\left(X ; B, B^{\prime}\right)
\end{gathered}
$$

which are defined in an analogous way as $\theta$. We can consider $B^{\prime}$ as a subset of $B$ by making the map $\pi$ an inclusion by homotopy, so the symbol $T(\gamma) / T\left(\gamma^{\prime}\right)$ makes sense. Also we define a map $l^{\prime}: H^{*}\left(B^{\prime}\right) \rightarrow H^{*}\left(B^{\prime}\right)$ $\otimes_{A} H^{*}(X)$ in an analogous way as $l$ was defined:

$$
l^{\prime}(x)=x \otimes_{A} c_{k} \otimes c_{n-k-q} .
$$

Let $I$ be the identity map on $H^{*}(X)$ and $i: B \rightarrow\left(B, B^{\prime}\right)$ be the inclusion 
then the following diagram is obviously commutative:

$$
\begin{array}{ccccc} 
& {\left[H^{*}\left(B, B^{\prime}\right) \otimes_{A} H^{*}(X)\right]^{n}} & \stackrel{\theta^{\prime \prime}}{\rightarrow} & \Omega_{n}^{*}\left(X ; B, B^{\prime}\right) \\
& & \downarrow i^{*} \otimes_{A} I & & \downarrow \Omega^{*}(i) \\
H^{q}(B) & \stackrel{l}{\rightarrow} & {\left[H^{*}(B) \otimes_{A} H^{*}(X)\right]^{n}} & \stackrel{\theta}{\rightarrow} & \Omega_{n}^{*}(X ; B) \\
\downarrow \pi^{*} & & & & \\
& & & & \downarrow \pi^{*} \otimes_{A} I \\
H^{q}\left(B^{\prime}\right) & \stackrel{l^{\prime}}{\rightarrow} & {\left[H^{*}\left(B^{\prime}\right) \otimes_{A} H^{*}(X)\right]^{n}} & \stackrel{\theta^{\prime}}{\rightarrow} & \Omega_{n}^{*}\left(X ; B^{\prime}\right)
\end{array}
$$

Clearly $\Omega(i), \Omega(\pi)$ are the maps induced by $i, \pi$ respectively at the bordism level and $\Omega^{*}(i), \Omega^{*}(\pi)$ their dual. From now on we will refer to the above diagram as diagram (1.2).

The next lemma reduces the computation of $I_{n}^{q}(B, S, p, k)$ to a homotopy problem.

LEMMA 1.3. $I_{n}^{q}(B, S, p, k)=\operatorname{Ker}\left(\Omega^{*}(\pi) \cdot \theta \cdot l\right)$.

Proof. The same as Lemma 2.2 of [2], p. 41.

Next we compute alg $I_{n}^{q}(B, S, p, k)$.

Proof of Theorem 0.7 (a). Let $(H, v)$ be an $n$-dimensional $B$-S Poincaré algebra (see Definition 0.3). Let $x \in H^{q}(B)$ such that $l(x) \in \operatorname{im}\left(i_{[S]} \otimes_{A} I\right)$, we will prove that $\nu(x)=0$. By Poincare duality it is enough to prove that $m_{H}(\nu(x) \cdot y \cdot z)=0$ for all $y \in H^{k}$ and all $z \in H^{n-k-q}$. Really, there is a map $f: H^{*}(X) \rightarrow H$ such that $f\left(c_{k}\right)=y, f\left(c_{n-k-q}\right)=z$, and $f$ is a map of graded algebras and left $A$ modules. This follows easily from the structure of $H^{*}(X)$ (see [6, p. 707]). So we get a map

$$
\nu \otimes_{A} f: H^{*}(B) \otimes_{A} H^{*}(X) \rightarrow H
$$

defined by $\nu \otimes_{A} f(a \otimes b)=\nu(a) \cdot f(b)$ for $a \in H^{*}(B), b \in H^{*}(X)$. Since $\nu(S)=\{0\}$ clearly $\nu([S])=\{0\}$ and so

$$
\left(\nu \otimes_{A} f\right) \cdot\left(i_{[s]} \otimes_{A} I\right)=0 .
$$

On the other hand $\left(\nu \otimes_{A} f\right)(l(x))=\nu(x) \cdot y \cdot z$ and because $l(x) \in \operatorname{im}\left(i_{[s]}\right.$ $\left.\otimes_{A} I\right)$, it follows from the previous remark that $\nu(x) \cdot y \cdot z=0$. So $\nu(x)=0$ and that ends the first half of the proof.

Next we will prove the converse. Let $x \in H^{q}(B)$ such that $l(x) \notin \operatorname{im}\left(i_{[S]}\right.$ $\left.\otimes_{A} I\right)$. Then we are going to construct an $n$-dimensional Poincaré algebra $H$ and a map $\nu: H^{*}(B) \rightarrow H$ of algebras and left-right $A$ modules such that $\nu(S)=\{0\}$ and $\nu(x) \neq 0$.

Let $R: H^{*}(B) \otimes H^{*}(X) \rightarrow H^{*}(B) \otimes_{A} H^{*}(X)$ be the natural quotient map. $H^{*}(B) \otimes H^{*}(X)$ which is an algebra and a left module over $A$, can be given a right $A$ module structure as follows: Let $\psi, \chi$ be the diagonal map and the canonical antihomomorphism of $A$ (see [8, p. 150, p. 166]), let $x \in H^{*}(B)$, $y \in H^{*}(X), a \in A$ and $\psi(a)=\sum a^{\prime} \otimes a^{\prime \prime}$, then we define 


$$
(x \otimes y) a=\sum\left(x a^{\prime}\right) \otimes\left(\chi\left(a^{\prime \prime}\right) y\right) .
$$

It can be proved that this formula is valid for all $x, y \in H^{*}(B) \otimes H^{*}(X)$ (see $\left[2\right.$, p. 46, Lemma 6.1 and p. 48, Lemma 7.1]). Let $F=\left(H^{*}(B) \otimes H^{*}(X)\right) \bar{A}$, where $\bar{A}$ is the set of elements of positive degree. We first note that $F \subseteq$ $\operatorname{kernel}(R)$. The reason is the following:

$$
\begin{aligned}
R((x \otimes y) a) & =R\left(\sum\left(x a^{\prime}\right) \otimes \chi\left(a^{\prime \prime}\right) y\right)=\sum\left(x a^{\prime}\right) \otimes_{A} \chi\left(a^{\prime \prime}\right) y \\
& =\sum x \otimes_{A} a^{\prime} \chi\left(a^{\prime \prime}\right) y=0,
\end{aligned}
$$

for $x \in H^{*}(B), y \in H^{*}(X), a \in \bar{A}, \psi(a)=\Sigma a^{\prime} \otimes a^{\prime \prime}$, because $\Sigma a^{\prime} \chi\left(a^{\prime \prime}\right)=0$ (see [8, p. 166]).

Next let us suppose that $x \in H^{q}(B)$ and $l(x) \notin \operatorname{im}\left(i_{[S]} \otimes_{A} I\right)$, this implies that $R(x \otimes 1) \neq 0$. Let $U=H^{*}(B) \otimes H^{*}(X)$ and let $z=U^{n *}=$ $\operatorname{Hom}\left(U^{n}, Z_{p}\right)$ be such that $z($ kernel $R)=\{0\}, z(l(x)) \neq 0$ and $z \cdot\left(i_{[S]} \otimes_{A} I\right)$ $=0$.

Let $J=\left\{y \in U^{i}: z\left(y \cdot U^{n-i}\right)=\{0\}, i \geqslant 0\right\}, J$ is clearly an ideal of $U$. We show that if $y \in J$ then $\chi(a) y \in J$ by induction on degree of $a$. For $a \in A_{0}$ this is true by assumption. Let $\operatorname{dim} a=i, \operatorname{dim} y=j$ and let $u \in U^{n-i-j}$. As noted above (see (1.4)), $u \cdot \chi(a) y=(u \cdot y) a-\Sigma\left(u \cdot a^{\prime}\right) \cdot \chi\left(a^{\prime \prime}\right) y$ where $\psi(a)$ $=1 \otimes a+\Sigma a^{\prime} \otimes a^{\prime \prime}$, and $\operatorname{dim} a^{\prime \prime}<i$. Hence by the inductive assumption and the fact that $z(F)=\{0\}$, we have $z(u \cdot \chi(a) y)=0$, so $\chi(a) y \in J$ and so $J$ is a left $A$-module. Let $H=U / J$ and let $r: U \rightarrow H$ be the projection. Let $\nu$ : $H^{*}(B) \rightarrow H$ be the map defined by $\nu(y)=r(y \otimes 1)$ and let $m_{H} \in\left(H^{n}\right)^{*}$ be defined by $m_{H} r=z$. Then $H$ is an unstable left algebra over $A$, because $J$ is an ideal closed under the left action of $A . H$ satisfies Poincare duality by construction, and $H^{i}=0$ for $i<0$ or $i>n$. Obviously $\nu(S)=\{0\}$ and $\nu(x) \neq 0$, so it remains to prove that $\nu$ is a map of right $A$ modules. But this means that

$$
m_{H}(\nu(y a) \cdot r(u))=m_{H}(\nu(y) \cdot \operatorname{ar}(u))
$$

for all $y \in H^{*}(B), a \in A, u \in U$. But this reduces to $z((y \otimes 1) a \cdot u)=z((y$ $\otimes 1) \cdot a u)$ which is true since $z(\operatorname{Ker} R)=\{0\}$. This concludes the proof of Theorem 0.7(a).

Next we turn our attention to the proof of Theorem 0.7(b), but first we need some preparation. Basically we need some results of $\mathrm{W}$. Browder about the homotopy type of $T(\gamma) / T\left(\gamma^{\prime}\right)$ (see [5]). Let $m$ be the dimension of the bundle $\gamma$, we assume $m>4 n$, and $d$ be the smallest dimension of elements of $S$. Let $v_{i} \in H^{i}(B)$ be the class defined by the relation $v_{i} U=\chi\left(P^{i}\right) U, i>0$, $v_{i}$ will be called the $i$ th Wu class of $\gamma$. The main technical result that we need for Theorem $0.7(\mathrm{~b})$ is the following.

Proposition 1.5. If no Wu class of $\gamma$ is a linear combination of elements of 
$S$, there is a map $r: T(\gamma) / T\left(\gamma^{\prime}\right) \rightarrow \Pi K\left(Z_{p}, *\right)$, from the space $T(\gamma) / T\left(\gamma^{\prime}\right)$ to a product of $K\left(Z_{p}, *\right)^{\prime} s$ which is an isomorphism in $Z_{p}$ cohomology up to dimension $m+2 d$.

The above Proposition is a slight variation of a result of $\mathrm{W}$. Browder (see [5, Theorem 6.1, Theorem 6.2]), we postpone its proof until the end of this section.

Proof of Theorem 0.7(b). In a manifold $M^{n}$ the Wu classes of the normal bundle, of dimension greater than $n / 2$ are zero (see [5, Proposition 2.3]), so we lose nothing by assuming that no $\mathrm{Wu}$ class is a linear combination of elements of $S$.

By the previous proposition the group $\Omega_{n}\left(X ; B, B^{\prime}\right)=\pi_{n+m}\left(T(\gamma) / T\left(\gamma^{\prime}\right)\right.$ $\left.\wedge X^{+}\right)$is a direct sum of $Z_{p}^{\prime}$ 's, and since $n+m<2 d+m$ the map $\theta^{\prime \prime}$ : $\left[H^{*}\left(B, B^{\prime}\right) \otimes_{A} H^{*}(X)\right]^{n} \rightarrow \Omega_{n}^{*}\left(X ; B, B^{\prime}\right)$ is an isomorphism (see [2, Lemma 3.2], the basic ingredient in the proof is that $T(\gamma) / T\left(\gamma^{\prime}\right)$ is a product of $K\left(Z_{p}, *\right)$ 's in the appropriate range of dimensions). On the other hand since the sequence $\Omega_{n}\left(X ; B^{\prime}\right) \rightarrow \Omega_{n}(X ; B) \rightarrow \Omega_{n}\left(X ; B, B^{\prime}\right)$ is exact, taking Hom $\left(, Z_{p}\right)$ we get an exact sequence $\Omega_{n}^{*}\left(X ; B, B^{\prime}\right) \rightarrow \Omega_{n}^{*}(X ; B) \rightarrow \Omega_{n}^{*}\left(X ; B^{\prime}\right)$. So taking into consideration Lemma 1.3, and the commutative diagram (1.2), we have: $x \in I_{n}^{q}(B, S, p, 0)$ means that $\Omega^{*}(\pi)(\theta(l(x)))=0$, or that $\theta l(x) \in$ im $\Omega^{*}(i)$, or that $\theta l(x) \in \operatorname{im}\left(\theta^{\prime \prime} \cdot \Omega^{*}(i)\right)=\operatorname{im} \theta \cdot\left(i^{*} \otimes_{A} I\right)$, which means that there is an element $y \in \operatorname{im}\left(i^{*} \otimes_{A} I\right)$ such that $\theta(y-l(x))=0$. But by an easy spectral sequence argument on the fibration $\pi: B^{\prime} \rightarrow B$ we can prove that image $\left(i^{*}\right)=[S]$, and so $\operatorname{im}\left(i^{*} \otimes_{A} I\right)$ is generated additively by elements of the form $a \otimes_{A} b$, where $a \in[S], b \in H^{*}(X)$. Notice that since we are examining the situation where $k=0, X=K\left(Z_{p}, n-q\right)$. But then $\operatorname{dim} a \geqslant d$ and $\operatorname{dim} a+\operatorname{dim} b=n$ so $\operatorname{dim} b \leqslant n-d$, and because of our dimensional assumptions in the statement of Theorem 0.7 (b) we have $\operatorname{dim} b<2(n-q)$ if $p$ is odd, and $\operatorname{dim} b<2(n-q)+1$ when $p=2$, which means (see [6, $\mathrm{p}$. 707]) that $b=t c_{n-q}$, for $t \in A$ and so $a \otimes_{A} b=a t \otimes_{A} c_{n-q}=l(a t)$. So the relation $\theta(y-l(x))=0$ because $\theta l(a t-x)=0$ which means that $a t-x \in$ $I_{n}^{q}(B, \varnothing, p, 0)$ and since $d \in[S], x \in I_{n}^{q}(B, \varnothing, p, 0)+[S]$, which ends the proof.

We conclude this section with the proof of Proposition 1.5.

Proof of Proposition 1.5. From now on in this paper, whenever $x$ is a cohomology class $|x|$ will be its dimension. Let $Z=\prod_{s \in S} K\left(Z_{p},|s|\right)$, then $\mathrm{W}$. Browder proved (see [5,Theorem 6.1]) that there is a map $h: T(\gamma) / T\left(\gamma^{\prime}\right) \rightarrow$ $T(\gamma) \wedge Z$ which is an isomorphism in $Z_{p}$ cohomology up to dimension $m+2 d-1$, and in dimension $m+2 d$ the kernel is generated by the elements $\left(c_{|s|}^{2}+c_{|s|} \cdot s\right) U$, where $s$ ranges over all $s \in S$ with $|s|=d$. Recall that $m$ is the dimension of the bundle $\gamma, U \in H^{m}(T(\gamma))$ its Thom class, and $c_{|s|}$ is the fundamental class of $K\left(Z_{p},|s|\right)$. 
To simplify the exposition we assume that $p=2$. The case where $p$ is odd is proved similarly (it is even simpler). Let $S^{\prime}$ be a $Z_{2}$ basis of $H^{*}(T(\gamma))$ which contains $\left\{\left(v_{d}+s\right) U\right\}$ where $s \in S$ and $|s|=d$, this is possible since no linear combination of elements of $S$ contains a Wu class. We call $S_{d}$ the subset of $S$ which consists of classes of dimension $d$. Let $Y=\Pi K\left(Z_{2},|s|+\left|s^{\prime}\right|\right)$ where $\Pi$ ranges over all couples $\left(s, s^{\prime}\right) \in S \times S^{\prime}, Y^{\prime}=\Pi K\left(Z_{2},|s|+\left|s^{\prime}\right|\right)$ where $\Pi$ ranges over all couples $\left(s, s^{\prime}\right) \in S \times S^{\prime}-S_{d} \times S^{\prime}$, and finally let $g: Y \rightarrow Y^{\prime}$ the obvious projection. According to W. Browder (see [5, Theorem 6.2']). there is a map $t: T(\gamma) \wedge Z \rightarrow Y$ such that $t^{*}\left(c_{|s|+\left|s^{\prime}\right|}\right)=s^{\prime} \otimes c_{|s|}$, which is an isomorphism in $Z_{2}$ cohomology up to dimension $m+2 d$. Next let us consider the elements in the kernel of $h_{m+2 d}^{*}$,

$$
\begin{aligned}
\left(c_{|s|}^{2}+c_{|s|} \cdot s\right) & U=\mathrm{Sq}^{|s|} c_{|s|}+c_{|s|} \cdot s \cdot U \\
= & c_{|s|} \cdot \chi\left(\mathrm{Sq}^{|s|}\right) U+\sum_{i=1}^{|s|} \mathrm{Sq}^{i}\left(c_{|s|} \cdot \chi\left(\mathrm{Sq}^{|s|-i}\right) U\right)+c_{|s|} \cdot s \cdot U \\
= & \left(v_{d}+s\right) U \otimes c_{|s|}+\sum_{i=1}^{|s|} \mathrm{Sq}^{i}\left(c_{|s|} \cdot \chi\left(\mathrm{Sq}^{|s|-i}\right) U\right)
\end{aligned}
$$

(see [10, Lemma 1]). This computation shows that the map $g t h: T(\gamma) / T\left(\gamma^{\prime}\right)$ $\rightarrow Y^{\prime}$ is an isomorphism in $Z_{2}$ cohomology up to dimension $m+2 d-1$, and there is no kernel in dimension $m+2 d$. So $T(\gamma) / T\left(\gamma^{\prime}\right)$ is a product of $K\left(Z_{2}, *\right)$ 's up to dimension $m+2 d$.

2. $B O$. In this section we will prove Theorem 0.8 for the case $B=B O$, but first we need a technical lemma.

LEMMA 2.1. Let $a \in A$ then $a c_{n-q}=0$ if and only if $a\left(c_{k} \otimes c_{n-k-q}\right)=0$.

Proof. For simplicity we will prove the lemma for the case $p=2$. The same proof with slight notational modifications is valid for any prime $p$. Actually we are going to use this result for the case where $p$ is an odd prime, in the next section.

Before proceeding with the proof of our lemma let us replace the notation with a more convenient one. We consider the Eilenberg-Mac Lane spaces $K\left(Z_{2}, m\right), K\left(Z_{2}, n\right)$ and let $a, b$ be their fundamental classes respectively. For this proof only $m, n$ are nonnegative integers, and they do not have the meaning that they had in the rest of this paper. A sequence $I=$ $\left(i_{1}, i_{2}, \ldots, i_{k}\right)$ is called admissible if $i_{1}, i_{2}, \ldots, i_{k}$ are nonnegative integers and $i_{1}>2 i_{2}, i_{2}>2 i_{3}, \ldots, i_{k-1}>2 i_{k}$. For an admissible sequence $I=$ $\left(i_{1}, \ldots, i_{k}\right)$ we define the excess $e(I)=i_{1}-i_{2}-i_{3}-\cdots-i_{k}$. So our lemma comes down to prove that the set of elements $\left\{\operatorname{Sq}^{I}(a \times b)\right\}$, where $I$ ranges over all admissible sequences $I$ such that $e(I)<m+n$ is linearly 
independent (see [6, p. 707]). We define $\mathrm{Sq}^{I}=\mathrm{Sq}^{i^{i}} \mathrm{Sq}^{i_{2}} \cdots \mathrm{Sq}^{i_{k}}$ and $d(I)=i_{1}$ $+i_{2}+\cdots+i_{k}$.

The proof will go by an induction on $n$, the inductive step can be proved by using the commutative diagram

$$
\begin{array}{ccc}
K\left(Z_{2}, m\right) \times K\left(Z_{2}, n+1\right) & \rightarrow & K\left(Z_{2}, n+m+1\right) \\
\downarrow & & \downarrow \\
K\left(Z_{2}, m\right) \times K\left(Z_{2}, n\right) \times K\left(Z_{2}, 1\right) & \rightarrow & K\left(Z_{2}, n+m\right) \times K\left(Z_{2}, 1\right)
\end{array}
$$

where the maps are defined in the obvious way.

So we assume that $n=1$. First we observe that if $I$ is an admissible sequence

$$
\mathrm{Sq}^{I}(a \times b)=\mathrm{Sq}^{I} a \times b+\sum \mathrm{Sq}^{I^{\prime}} a \times \mathrm{Sq} I^{\prime \prime} b
$$

where $d\left(I^{\prime}\right)<d(I)$.

Now let us suppose that $e(I)=m+1$, for an admissible sequence $I$, this means that $I=\left(2^{l-1} i_{l}, 2^{l-2} i_{l}, \ldots, 2 i_{l}, i_{l}, i_{l+1}, \ldots, i_{k}\right)$ where $i_{l}>2 i_{l+1}$ and $i_{l}=i_{l+1}+i_{l+2}+\cdots+i_{k}+(m+1)$. Let $J=\left(i_{l+1}, \ldots, i_{k}\right)$, clearly $e(J)<$ $m+1$ and $\mathrm{Sq}^{I}(a \times b)=\left(\mathrm{Sq}^{J}(a \times b)\right)^{2^{I}}=\left(\mathrm{Sq}^{J} a\right)^{2^{\prime}} \times b^{2^{l}}+\Sigma\left(\mathrm{Sq}^{I^{\prime}} a\right)^{2^{\prime}} \times$ $\left(\mathrm{Sq}^{I \prime \prime} b\right)^{2^{\prime}}$ where $d\left(I^{\prime}\right)<d(J)$.

On the other hand if $e(I)<m+1$ we have $\mathrm{Sq}^{I}(a \times b)=\mathrm{Sq}^{I} a \times b+$ $\Sigma \mathrm{Sq}^{I^{\prime}} a \times \mathrm{Sq}^{I^{\prime \prime}} b$ where $d\left(I^{\prime}\right)<d(I)$.

But the set of elements $\left(\mathrm{Sq}^{I} a \times b\right)^{2^{\prime}}$ for $l \geqslant 0 e(I)<m+1$ are linearly independent, and so the set of elements $\mathrm{Sq}^{I}(a \times b)$ for $e(I)<m+1$ are linearly independent.

Proof of TheOREM 0.8, For $B=B O$. (a) In the case when $B=B O$, the map $\theta$ is an isomorphism (see [2, p. 42, Lemma 3.2]), so from the diagram (1.2) and Lemma 1.3 we have that $x \in I_{n}^{q}(B O, S, 2, k)$ means $\theta l(x) \in$ $\operatorname{im}\left(\Omega^{*}(i)\right)$, but since $\theta^{\prime \prime}$ is an isomorphism we get that

$$
l(x) \in \operatorname{image}\left(i^{*} \otimes_{A} I\right)=\operatorname{im}\left(i^{*}[s] \otimes_{A} I\right)
$$

which by Theorem 0.7 (a) means that $x \in \operatorname{alg} I_{n}^{q}(B O, S, 2, k)$.

(b) It is known that $H^{*}(B O)$ is a free right module over $A$ (see [2, p. 42]), let $\left(x_{i}\right)$ be a basis over $A$, and let $x \in \sum x_{i} a_{i}, a_{i} \in A$, then $x \in I_{n}^{q}(B O, \varnothing, 2, k)$ if and only if $a_{i}\left(c_{k} \otimes c_{n-k-q}\right)=0$ and $x \in I_{n}^{q}(B O, \varnothing, 2,0)$ if and only if $a_{i} c_{n-q}=0$, which, according to the previous lemma, means the same thing

3. $B S O$ and $B U$. Throughout this section $B$ will be $B S O$ or $B U$, and $p$ will be an odd prime whenever $B=B S O$. We are going to prove Theorem 0.8 for the case $B=B S O$ or $B U$. Basically we exploit the technique of [3]. The main goal of this section is to prove that $\theta$ restricted over a certain subset of $H^{*}(B) \otimes_{A} H^{*}(X)$ is a monomorphism (see Theorem 3.10 below), this will be accomplished by constructing a sufficient number of elements of $\Omega_{*}(X ; B)$. 
We start by quoting a few well-known results about the Steenrod algebra $A$. When $p=2$ we denote $\mathrm{Sq}^{2 i}$ by $P^{i}$ and $S q^{1}$ by $\delta$. Let $A^{\prime} \subset A$ be the submodule generated by all $P^{I}$ where $I$ is an admissible sequence (see $\$ 2$, Lemma 2.1). In case $p$ is odd $A^{\prime}$ is a subalgebra, and in general $A^{\prime}$ is naturally isomorphic to $A$ mod the two sided ideal $(\delta)$ generated by the Bochstein $\delta$.

Definition 3.1. A sequence $R=\left(r_{1}, r_{2}, \ldots\right)$ will be called a sequence of numbers if and only if $r_{1}, r_{2}, \ldots$ are nonnegative numbers, all of which are zero except a finite number. When $R$ is a sequence of numbers we put $n(R)=\Sigma 2 r_{i}\left(p^{i}-1\right) l(R)=\Sigma r_{i}$.

In [8, p. 162], Milnor defined an element $P^{R} \in A^{\prime}$ for each sequence of numbers $R$ and showed that $\left\{P^{R}\right\}$ is a basis for $A^{\prime}$. Note that $P^{I}$ and $P^{R}$ are different things. The reader should be cautioned that when $p=2, P^{R}$ is what Milnor denotes $\mathrm{Sq}^{2 R}$, where $2 R=\left(2 r_{1}, 2 r_{2}, \ldots\right)$ (see also [9, p. 507]).

Next we construct some elements of $\Omega_{*}(X ; B)$ where $B=B U$ or $B S O$. All manifolds will be assumed oriented. If $Y$ is a space $Y^{m}$ will be the $m$-fold cartesian product and if $Y$ is a manifold [ $Y$ ] will be the generator of the top cohomology.

For each sequence of numbers $R=\left(r_{1}, r_{2}, \ldots\right)$ let $M_{R}=\Pi\left(\left(C P^{p^{\prime}}\right)^{r_{i}}\right.$ X $\left(S^{1}\right)^{k-2 l(R)}$.

Note. $\operatorname{dim} M_{R}=k+n(R)$. Let $u_{R} \in H^{*}\left(M_{R}\right)$ be the tensor product of the ring generators of each factor. We quote from $[3$, p. 359]

LEMMA 3.2. If $n\left(R^{\prime}\right) \geqslant n(R)$

$$
\begin{aligned}
P^{R^{\prime}} u_{R} & =0 \quad \text { if } R \neq R^{\prime}, \\
& =\left[M_{R}\right] \text { if } R=R^{\prime} .
\end{aligned}
$$

DEFinition 3.3. A sequence of sequences $L=\left(R_{1}, R_{2}, \ldots, R_{m}\right)$ is a finite sequence of sequences of numbers, decreasing in the lexicographic order, such that no sequence of numbers appears more than $(p-1)$ times.

We define $M_{L}=\Pi M_{R_{i}}, u_{L}=\sum u_{R_{i}}$. If $x$ is a cohomology class,

$$
P^{L} x=P^{R_{1}} x P^{R_{2}} \cdots P^{R_{m} x} \text {. }
$$

The next lemma is the main technical result before the proof of Theorem 3.10 .

LEMMA 3.4. If $\operatorname{dim}\left(P^{L^{\prime}} u_{L}\right) \geqslant \operatorname{dim}\left(P^{L} u_{L}\right)$,

$$
\begin{aligned}
P^{L^{\prime} u_{L}}=0 & \text { if } L^{\prime} \neq L, \\
& \neq 0 \quad \text { if } L^{\prime}=L .
\end{aligned}
$$

The above result plays in this paper the same role that Lemma 3.2 plays in [3]. We will split its proof to four sublemmas. 
LeMma 3.5. If $L=\left(R_{1}, \ldots, R_{m}\right)$ and $R_{1}=R_{2}=\cdots=R_{m}$ then $P^{L} u_{L}=$ $\pm m !\left[M_{L}\right]$

Proof. By Lemma 3.2 and Definition 3.3, $P^{L} u_{L}=\left(\Sigma\left[M_{R_{1}}\right)^{m}= \pm \Pi\left[M_{R_{1}}\right]\right.$ $= \pm m !\left[M_{L}\right]$.

LeMma 3.6. If $L=\left(R_{1}, \ldots, R_{m}\right), L^{\prime}=\left(R_{1}, \ldots, R_{l}\right), l>m$ and $R_{1}=R_{2}$ $=\cdots=R_{l}$ then $P^{L^{\prime}} u_{L}=0$.

PROOF. $P^{L^{\prime} u_{L}}=\left(\sum_{i=1}^{m}\left[M_{R_{R}}\right]\right)^{l}=0$.

Lemma 3.7. Let $L=\left(R_{1}, R_{2}, \ldots, R_{m}\right)$ and let $g(1), g(2), \ldots, g(m)$ be a finite sequence from the numbers $1,2, \ldots, m$, then if $\Pi P^{R_{1} u_{R_{g(0)}}} \neq 0$ then

$$
\operatorname{dim} P^{R_{i}} u_{g(i)}=\operatorname{dim} M_{R_{g}(i)} \text { for } i=1,2, \ldots, m .
$$

Proof. Since $\operatorname{dim}\left(\Pi \mathrm{P}^{R_{i}} u_{R_{8(0)}}\right)=\operatorname{dim} M_{L}=\Sigma \operatorname{dim} M_{R_{i}}$, the proof is obvious.

LEMMA 3.8. $P^{L} u_{L} \neq 0$.

PRoof. $P^{L} u_{L}=\Pi_{i}\left(\sum_{j} P^{R u_{R j}}\right)$, combining Lemmas 3.2, 3.5, 3.7, the result follows easily.

Proof of Lemma 3.4. The case $L=L^{\prime}$ has already been settled in Lemma 3.8. We can dispose very easily of the case where $\left(\operatorname{dim} P^{L^{\prime}} u_{L}\right)>\operatorname{dim} P^{L^{L} u_{L} \text {. So }}$ let us suppose that $\operatorname{dim} P^{L^{\prime}} u_{L}=\operatorname{dim} P^{L_{u_{L}}}$ and $L^{\prime} \neq L$. Let $L=$ $\left(R_{1}, \ldots, R_{m}\right)$ and $L^{\prime}=\left(R_{1}^{\prime}, \ldots, R_{l}^{\prime}\right)$, then

$$
P^{L^{\prime} u_{L}}=\prod_{i=1}^{l}\left(\sum_{j=1}^{m} P^{R_{i}^{\prime} u_{R_{j}}}\right) \text {. }
$$

If $P^{L^{\prime}} u_{L} \neq 0$ by Lemma 3.7 we must have $l=m$, furthermore by Lemma 3.2 $L$ and $L^{\prime}$ should include the same sequences of numbers, but since $L^{\prime} \neq L, L^{\prime}$ must include one sequence of numbers more times than the same sequence appears in $L$, and by Lemma 3.6, $P^{L^{\prime}} u_{L}=0$.

The definition of $M_{R}, u_{R}, M_{L}, u_{L}$ depends on $k$, we could give the same definition with $n-k-q$ instead and call the corresponding objects $M_{R}^{\prime}, u_{R}^{\prime}, M_{L}^{\prime}, u_{L}^{\prime}$, and the analogous results of Lemmas 3.2, 3.4, 3.5, 3.6, 3.7, 3.8 are equally valid. Now we have in our hands all the technical tools to translate all the results of [3] in our situation.

Let $\pi^{\prime}(m)$ be the set of partitions of $m$ containing no integer of the form $\left(p^{i}-1\right) / 2$. Let $S_{\omega}$ be the corresponding element (see [9, p. 515]). Milnor [9] has proved the equivalent of the following:

THEOREM 3.9'. If $p$ is odd, $H^{*}(B S O)$ is a free right $A^{\prime}$ module, and $\left\{S_{\omega} \mid \omega \in \pi^{\prime}(m), m \geqslant 0\right\}$ is a basis. Furthermore for each $\omega \in \pi^{\prime}(m)$ there is an oriented $4 m$ manifold $N_{\omega}$ such that, if $\omega, \omega^{\prime} \in \pi^{\prime}(m)$, 


$$
\begin{aligned}
& \nu_{N_{\omega}}^{\prime}\left(S_{\omega^{\prime}}\right)\left(N_{\omega}\right)=0 \text { if } \omega^{\prime} \neq \omega \text {, } \\
& \neq 0 \text { if } \omega^{\prime}=\omega \text {, }
\end{aligned}
$$

where $\nu_{N_{\omega}}$ is the map which classifies the stable normal bundle of $N_{\omega}$.

Just for the next theorem we assume that $k, n-k-q$ are even numbers.

THeOREM 3.10. If $p$ is odd, $\left\{\theta\left(S_{\omega} \otimes_{A} P^{L} c_{k} \otimes P^{L^{\prime}} c_{n-k-q}\right): \omega \in \pi^{\prime}(m), m>\right.$ $0, L=\left(R_{1}, R_{2}, \ldots,\right), L^{\prime}=\left(R_{1}^{\prime}, R_{2}^{\prime}, \ldots,\right), l\left(R_{i}\right)<k \mid 2, l\left(R_{i}^{\prime}\right)<(n-k-$ $q) \mid 2\}$, where $\theta$ is the homomorphism described in diagram (1.2), forms a linearly independent set in $\Omega(X ; B S O)^{*}$.

Proof. Let $L, L^{\prime}$ be sequences of sequences (see Definition 3.3) and $\omega \in \pi^{\prime}(m)$, then define $\alpha\left(\omega, L, L^{\prime}\right)=\left\{N_{\omega} \times M_{L} \times M_{L^{\prime}}^{\prime}, p\left(u_{L} \times u_{L^{\prime}}^{\prime}\right)\right\}$ where $p: N_{\omega} \times M_{L} \times M_{L^{\prime}}^{\prime} \rightarrow M_{L} \times M_{L^{\prime}}^{\prime}$ is the projection and $u_{L} \times u_{L^{\prime}}^{\prime}: M_{L} \times M_{L^{\prime}}^{\prime}$ $\rightarrow K\left(Z_{p}, k\right) \times K\left(Z_{p}, n-k-q\right)$. And then the proof goes as Theorem 3.3 of [3].

REMARK. An analogous result holds for the cases that $k$ or $n-k-q$ are odd numbers. Specifically if let us say $k$ is odd then $L=\left(R_{1}, R_{2}, \ldots, R_{m}\right)$ ranges over all sequences of sequences such that the $R_{1}, R_{2}, \ldots, R_{m}$ are distinct, and the theorem holds.

Definition 3.11. Let $A^{\prime \prime}$ be the two sided ideal if $A$ generated by $\delta$ and $X^{\prime \prime}$ be the two sided ideal in $H^{*}(X)$ generated by $a c_{k}, a c_{n-k-q}$ for $a \in A^{\prime \prime}$. Let $X^{\prime}$ be the subalgebra of $H^{*}(X)$ generated by the elements $a c_{k}, a c_{n-k-q}, a \in A^{\prime}$. Clearly as $Z_{p}$ modules $A=A^{\prime} \oplus A^{\prime \prime}, H^{*}(X)=X^{\prime} \oplus X^{\prime \prime}$.

LemMa. Let $I_{X^{\prime}}, I_{X^{\prime \prime}}$ be the inclusions $X^{\prime} \subseteq H^{*}(X)$ and $X^{\prime \prime} \subseteq H^{*}(X)$ respectively, and $p$ an odd prime. Then suppose that $x \in \operatorname{image}\left(I_{A} \otimes_{A} I_{X^{\prime}}\right)$ and $y \in \operatorname{image}\left(I_{A} \otimes_{A} I_{X^{\prime \prime}}\right)$ and that $\theta(x+y)=0$ then $\theta(x)=\theta(y)=0$.

Proof. Obviously $\theta(y)\left(\alpha\left(\omega, L, L^{\prime}\right)\right)=0$ (see proof of Theorem 3.10) so $\theta(x)\left(\alpha\left(\omega, L, L^{\prime}\right)\right)=0$ and so by Theorem $3.10 \theta(x)=0$ and so $\theta(y)=0$.

Proof of Theorem 0.8, For $B=B S O, P$ OdD PRIME. (a) We argue as in the case $B=B O$. If $x \in I_{n}^{q}(B S O, S, p, k)$ then there is $y \in \operatorname{im}\left(i^{*} \otimes_{A} I\right)$ so that $\theta(l(x)-y)=0$ (see diagram (1.2)) but $y=y^{\prime}+y^{\prime \prime}$ where $y^{\prime} \in$ $\operatorname{im}\left(i^{*} \otimes_{A} I_{X^{\prime}}\right)$ and $y^{\prime \prime} \in \operatorname{im}\left(i^{*} \otimes_{A} I_{X^{\prime \prime}}\right)$ so $\theta\left(l(x)-y^{\prime}-y^{\prime \prime}\right)=0$ and by the previous lemma this means that $\theta\left(l(x)-y^{\prime}\right)=0$, and by Theorem 3.10 $l(x)-y^{\prime}=0$ which means $x \in \operatorname{alg} I_{n}^{q}(B S O, S, p, k)$.

(b) Let $x \in I_{n}^{q}(B S O, \varnothing, p, k)$, this means that $\theta l(x)=0$, which by Theorem 3.10 means $l(x)=0$. But by Theorem 3.9, $x=\Sigma c_{\omega, R} \cdot\left(S_{\omega}\right) P^{R}$ where $c_{\omega, R} \in Z_{p}$. Recall (see Lemma 2.1, and from [3, Theorem 2.4]) that $P^{R}\left(c_{k} \otimes c_{n-k-q}\right)=0$ if and only if $l(R)>(n-q) \mid 2$. Therefore 


$$
\begin{aligned}
0 & =\theta l(x) \\
& =\theta\left(x \otimes_{A} c_{k} \otimes c_{n-k-q}\right) \\
& =\sum c_{\omega, R} \theta\left[s_{\omega} \otimes_{A} P^{R}\left(c_{k} \otimes c_{n-k-q}\right)\right] \\
& =\sum_{l(R)<(n-q) / 2} c_{\omega, R} \theta\left[s_{\omega} \otimes_{A} P^{R}\left(c_{k} \otimes c_{n-k-q}\right)\right]
\end{aligned}
$$

but by Theorem $3.10\left\{\theta\left[S_{\omega} \otimes_{A} P^{R}\left(c_{k} \otimes c_{n-k-q}\right)\right]\right\}: l(R) \leqslant(n-q) / 2$ are linearly independent. Hence $c_{\omega, R}=0$ for $l(R) \leqslant(n-q) / 2$, but by Lemma 4.1 of [3] this means that $x \in I_{n}^{q}(B S O, \varnothing, p, 0)$, which completes the proof.

The proof of Theorem 0.8 for the case $B=B U$ follows exactly the same pattern. There is an analogue for Theorem 3.9 due to Milnor (see $[9$, p. 516]), and every subsequent theorem could be easily duplicated. I am sure that the reader who survived up to this point can provide the details.

\section{BIBLIOGRAPHY}

1. J. F. Adams, On formulae of Thom and $W u$, Proc. London Math. Soc. 11 (1961), 741-752.

2. Edgar H. Brown, Jr. and Franklin Peterson, Relations among characteristic classes. I, Topology 3 (1964), 39-52.

3. __ Relations among characteristic classes. II, Ann. of Math. (2) 81 (1965), 356-363.

4. __ Algebraic bordism groups, Ann. of Math. (2) 79 (1964), 616-622.

5. William Browder, The Kervaire invariant of framed manifolds and its generalizations, Ann. of Math. (2) 90 (1969), 157-186.

6. Henri Cartan, Sur les groupes d'Eilenberg-Mac Lane. II, Proc. Nat. Acad. Sci. U.S.A. 40 (1954), 704-707.

7. Richard Lashof, Poincaré duality and cobordism, Trans. Amer. Math. Soc. 109 (1963), 257-277.

8. John Milnor, The Steenrod algebra and its dual, Ann. of Math. (2) 67 (1958), 150-171.

9. - On the cobordism ring $\Omega^{*}$ and a complex analogue. I, Amer. J. Math. 82 (1960), 505-521.

10. S. Papastavridis, A formula for the obstruction to transversality, Topology 11 (1972), 415-416.

11. Robert E. Stong, Poincarea 3 algebras modulo an odd prime, Comment. Math. Helv. 49 (1974), 382-407.

12. 1968.

2.

13. René Thom, Quelques propriétés globales des variétés differentiables, Comment. Math. Helv. 28 (1954), 17-86.

14. W. Stephen Wilson, A new relation on the Stiefel-Whitney classes of spin manifolds, Illinois J. Math. 17 (1973), 115-127.

Department of Mathematics, Brandeis University, Waltham, Massachusetts 01254

Current address: Department of Mathematics, University of Crete, Iraklion, Greece 